Trauma Berufskrankh 2012 · 14[Suppl 3]:385-387 DOI 10.1007/s10039-012-1844-2

Online publiziert: 29. März 2012

(c) Springer-Verlag 2012

P.C. Strohm ${ }^{1}$ P.P. Schmittenbecher ${ }^{2}$

${ }^{1}$ Kindertraumatologie, Department Orthopädie und Traumatologie,

Klinikum der Albert-Ludwigs-Universität Freiburg

${ }^{2}$ Klinik für Kinderchirurgie, Städtisches Klinikum Karlsruhe

\title{
Cubitus varus/valgus nach Frakturen des distalen Humerus
}

\section{Indikationen zur Korrekturosteotomie}

Fehlstellungen nach Frakturen des distalen Humerus sind bekannte Probleme. Am häufigsten sind Rotationsfehler, es sind aber auch Fehlstellungen in der sagittalen oder frontalen Ebene möglich. Durch die primäre, regelrechte operative Stabilisierung der Frakturen kann die Zahl der Fehlstellungen deutlich verringert werden $[2,7]$.

Ein Cubitus varus stellt die häufigste Komplikation nach suprakondylären Frakturen des Humerus dar; seine Inzidenz wird mit $3-57 \%$ angegeben $[1,2,3]$. Ein Cubitus valgus wurde nach diesen Verletzungen dagegen selten beschrieben [6]. Beide Formen sind entweder Folge von belassenen Fehlstellungen oder sie treten nach konservativer oder operativer Behandlung mit ungenügender Reposition oder Retention auf. Dies betrifft vorwiegend die Frakturtypen III und IV nach von Laer et al. [5], die durch einen mehr oder minder ausgeprägten Rotationsfehler und hohe Instabilität gekennzeichnet sind. Bereits geringe Drehfehler reduzieren die Kontaktfläche der Fragmente erheblich, sekundär kommt es dann zur Verkippung in der Frontalebene. Sowohl ein Cubitus varus als auch ein Cubitus valgus können aber auch nach verschiedenen anderen Verletzungen des distalen Humerus durch unvollständige Reposition oder Wachstumsstörungen zustande kommen. In diesem Kontext muss an erster Stelle die Condylus-radialis-Fraktur erwähnt werden.

\section{Ätiologie und Epidemiologie}

Die meisten Fehlheilungen entstehen nach suprakondylären Humerusfrakturen, gefolgt von Kondylenfrakturen. Dabei stellt der Cubitus varus, wie oben angeführt, die häufigste Komplikation nach suprakondylären Humerusfrakturen dar. Mehr als 90\% der Fälle sind Folge von Extensionsfrakturen mit Kollaps der medialen Kondylensäule, weitere von ulnarem Minderwuchs nach Verschluss der ulnaren Wachstumsfuge und wenige von radialem Mehrwachstum nach radialen Kondylenfrakturen.

Der Cubitus valgus bildet sich nach den seltenen posterolateralen Extensionsfrakturen und den noch selteneren Flexionsfrakturen, jeweils durch lateralen Kollaps, in wenigen Fällen auch durch ulnares Mehrwachstum nach ulnaren Kondylenfrakturen.

\section{Allgemeines}

Die meisten Autoren gehen davon aus, dass geringe Spontankorrekturen noch bis etwa zum 7. Lebensjahr möglich sind, danach jedoch eigentlich nicht mehr erwartet und einkalkuliert werden können [6]. Spontankorrekturen erfolgen fast aus- schließlich in der sagittalen Ebene und können einen Antekurvationsfehler ausgleichen, kaum aber den Varus- oder Valgusfehler.

Die Probleme der Fehlstellungen können (häufig) kosmetischer Natur sein oder (selten) sich in Bewegungseinschränkungen äußern. Die Streckhemmung ist die häufigste Bewegungseinschränkung, gefolgt von der Beugehemmung (selten) und geminderten Rotationsfähigkeit (rar). In den meisten Fällen sind die Bewegungseinschränkungen funktionell von geringer bis gar keiner Bedeutung.

Als weitere Komplikation kann es durch die Fehlstellungen zu Nervenirritationen (N. ulnaris, N. radialis) kommen.

\section{Operation}

\section{Indikation}

Die Indikation zum operativen Korrekturversuch stellt in der Regel der Patient mit seinen Eltern. Gründe für die operative Korrektur können deutliche Bewegungseinschränkung, Pseudarthrose, vermeintliche Zunahme der Wachstumsstörung und auch kosmetische Gründe sein.

Grundsätzlich handelt es sich bei sämtlichen Korrekturen um rehabilitative Eingriffe. Das heißt, der Patient signalisiert und entscheidet, was benötigt wird. Eine Rehabilitation gegen die Interessen des 


\section{Übersichten}
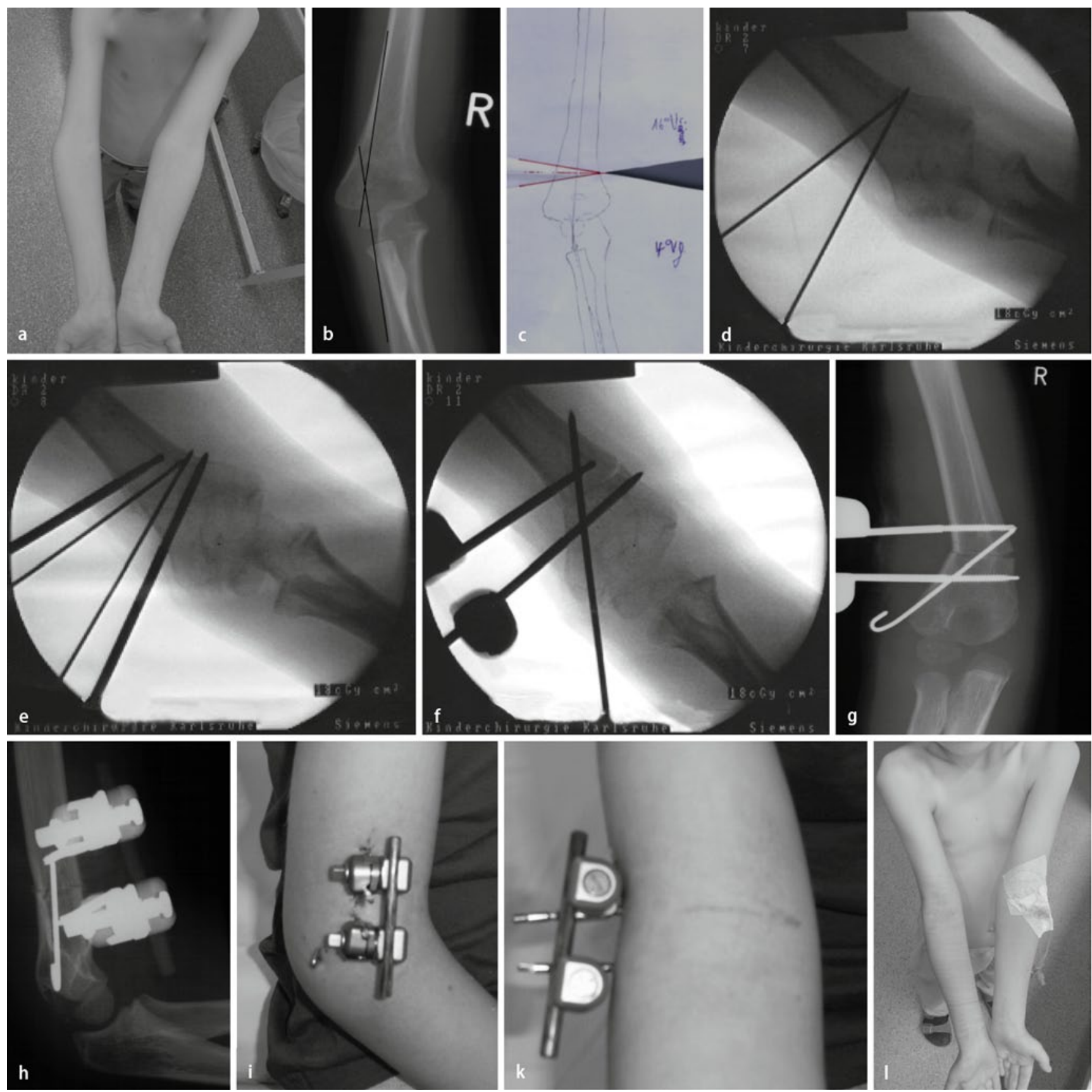

Abb. $1 \Delta$ 8-jähriger Junge nach suprakondylärer Humerusfraktur rechts mit a Cubitus varus von $16^{\circ}$ im Vergleich zur Gegenseite mit $4^{\circ}$, b Röntgenbild zur Operationsplanung, c Planungsskizze, $\mathbf{d}-\mathbf{f}$ intraoperative Aufnahmen, $\mathbf{d}$ nach Vorlegen der Drähte im geplanten Winkel, e nach Eindrehen der Schanz-Schrauben, $\mathbf{f}$ nach Osteotomie, Korrektur und zusätzlicher Sicherung mit Kirschner-Draht, $\mathbf{g , h}$ postoperatives Röntgen in 2 Ebenen, i,k postoperatives, klinisches Bild, I klinisches Ergebnis nach Materialentfernung 8 Wochen postoperativ

Patienten ist kontraproduktiv, da man bei sämtlichen Maßnahmen auf dessen Kooperation und Compliance angewiesen ist. Die Interessen des Patienten sind daher selbst gegenüber den Eltern zu vertreten und ggf. durchzusetzen und nicht umgekehrt [6].
Grundsätzlich sind zu jedem Zeitpunkt - unabhängig davon, ob die Wachstumsfugen noch offen sind oder nicht Korrektureingriffe möglich.

In den meisten Fällen handelt es sich beim Cubitus varus um eine rein kosmetische Deformität. Nur vereinzelt wurden funktionelle Komplikationen im Bereich des Ellenbogens, der Schulter oder des $\mathrm{N}$. ulnaris geschildert.

\section{Technik}

Für die Korrektureingriffe wurden die unterschiedlichsten Verfahren beschrieben. Einerseits können sie zwei- oder 
dreidimensional durchgeführt werden, und der Zugang kann von medial oder lateral gewählt werden. Es kommen sog. Open- und Closed-Wedge-Techniken zum Einsatz. Erstere werden mit oder ohne Augmentation durchgeführt.

Wie oben beschrieben ist eine Korrektur unabhängig vom Reifungszustand der Wachstumsfuge in jedem Alter mög lich. Zur Stabilisierung werden Platten, Kirschner-Drähte, Klammern und der Fixateur externe verwendet (• Abb. 1). Es sollten keine Minimalosteosynthesen durchgeführt werden, die eine anschließende Gipsruhigstellung erfordern, als Ziel der Maßnahme sollte folglich die Übungsstabilität gefordert werden.

Nach unserer Meinung und der anderer Autoren sollten bei der Korrektur sämtliche Komponenten in allen Ebenen des Raums korrigiert werden, um ein sicheres und kosmetisch befriedigendes Ergebnis zu erreichen [2,6]. Von den meisten Autoren wird eine zuklappende Osteotomie von lateral gewählt. Vorteil dieses Vorgehens ist einerseits, dass keine Augmentation notwendig ist, außerdem ist die Gefahr der Verletzung von Nerven geringer. Der N. radialis sollte jedoch dargestellt werden. Die durch die Closed-Wedge-Technik entstehende Verkürzung ist klinisch irrelevant. Eine bewegungsstabile Retention bei gleichzeitiger Korrekturmöglichkeit in sämtlichen Ebenen lässt sich am leichtesten und effektivsten mit einer externen Fixation erreichen. Dabei ist eine unilaterale Montage sehr gut geeignet. Die Plattenosteosynthese erlaubt keine intra- oder postoperativen Nachkorrekturen, zur Metallentfernung ist ein weiterer operativer Eingriff nötig [4].

Schlechte Ergebnisse nach suprakondylären Korrekturen werden in 15-43\% der Fälle angegeben, Komplikationen in bis zu $60 \%$. Dabei stehen Fehlstellungsrezidive im Vordergrund [6]. Auf diese Tatsache sollte beim Aufklärungsgespräch und der Indikationsstellung auf jeden Fall Rücksicht genommen werden.

\section{Fazit für die Praxis}

Die Indikation zur Operation ist streng zu stellen. Bei entsprechendem Leidens- druck und Operationswunsch ist die Korrektur jedoch in jedem Alter möglich. Es müssen auf jeden Fall die Interessen des Patienten selbst im Vordergrund stehen. Bei diesen Eingriffen handelt es sich selbstverständlich niemals um eine Notfallindikation. Zur Stabilisierung nach (mehrdimensionaler) Korrekturosteotomie ist der Fixateur externe als gutes und zuverlässiges Instrument bewährt.

\section{Korrespondenzadresse}

Prof. Dr. P.C. Strohm

Kindertraumatologie, Department Orthopädie und Traumatologie, Klinikum der Albert-Ludwigs-Universität Freiburg, Hugstetter Straße 55, 79106 Freiburg peter.strohm@uniklinik-freiburg.de

Interessenkonflikt. Der korrespondierende Autor gibt für sich und seine Koautoren an, dass kein Interessenkonflikt besteht.

The supplement containing this article is not sponsored by industry.

\section{Literatur}

1. Gaddy BC, Manske PR, Pruitt DI et al (1994) Distal humeral osteotomy for correction of posttraumatic cubitus varus. J Pediatr Orthop 14:214-219

2. Handelsman JE, Weinberg J, Hersch JC (2006) Corrective supracondylar humeral osteotomies using the small AO external fixator. J Pediatr Orthop B 15:194-197

3. Hernandez MA III, Roach JW (1994) Corrective osteotomy for cubitus varus deformity. J Pediatr Orthop 14:487-491

4. Laer $L$ von (2004) Korrektureingriffe am kindlichen Ober- und Unterarm. Unfallchirurg 107:552-562

5. Laer L von (1979) Die suprakondyläre Humerusfraktur im Kindesalter. Arch Orthop Trauma Surg 95:123-150

6. Linhart WE, Kraus T (2008) Reconstruction of humeroradial joint. Oper Orthop Traumatol 20:396408

7. Reising K, Schmal H, Kohr M et al (2011) Surgical treatment of supracondylar humerus fractures in children. Acta Chir Orthop Traumatol Cech 78:519523
Trauma Berufskrankh 2012 • 14[Suppl 3]:385-387

DOI 10.1007/s10039-012-1844-2

(C) Springer-Verlag 2012

\section{P.C. Strohm · P.P. Schmittenbecher \\ Cubitus varus/valgus nach \\ Frakturen des distalen \\ Humerus. Indikationen zur Korrekturosteotomie}

\section{Zusammenfassung}

Fehlstellungen nach Frakturen des distalen Humerus im Kindesalter werden immer wieder beobachtet. Am häufigsten kommt es zu einem Cubitus varus. Durch primär regelrechte Therapie kann die Rate an posttraumatischen Fehlstellungen deutlich reduziert werden. Klinisch sind in der Regel geringe Einschränkungen vorhanden, trotzdem besteht in bestimmten Fällen die Indikation zur Korrekturosteotomie. Geeignet erscheinen uns dann mehrdimensionale Korrekturen, welche mit dem Fixateur externe übungsstabil retiniert werden.

\section{Schlüsselwörter}

Cubitus varus · Komplikationen · Humerus . Kinder $\cdot$ Osteotomie

\section{Cubitus varus/valgus following distal humeral fractures. Indications for corrective osteotomies}

\section{Abstract}

The surgical correction of malunited distal humeral fractures in children is a challenging procedure for the orthopedic surgeon. The best prevention is correct treatment of the initial fracture. When correction is required we recommend a multidirectional osteotomy with stabilization by an external fixator. Indications can be for cosmetic purposes, pseudarthrosis, and limited movement of the elbow joint and the forearm.

\section{Keywords}

Cubitus varus $\cdot$ Complications $\cdot$ Humerus . Children · Osteotomy 